\title{
The cannabinoid receptor CB1 affects the proliferation and apoptosis of adenomyotic human uterine smooth muscle cells of the junctional zone: a mechanism study
}

\author{
Sha Wang, Bohan Li, Xue Shen, Hua Duan*, Zhengchen Guo, Xiao Li and Fuqing Sun
}

\begin{abstract}
Background: The denomyotic junctional zone (JZ) plays an important role in the pathogenesis of adenomyosis. Proliferating cell nuclear antigen (PCNA) is an important nuclear marker of cell proliferation. This study aimed to evaluate the effects of the cannabinoid receptor CB1 on proliferation and apoptosis in the JZ in women with and without adenomyosis.
\end{abstract}

Methods: JZ smooth muscle cells (JZSMCs) of the adenomyosis and control groups were collected and cultivated. Immunohistochemistry and immunoblotting were used for protein localization and expression detection of CB1 and PCNA. Additionally, qRT-PCR was used to quantitatively analyse the mRNA expression of the two. AM251 and ACEA were used to regulate the function of CB1 receptors, and CCK-8 assay and flow cytometry assay were used to verify the proliferation and apoptosis of JZSMCs after regulation.

Results: We demonstrated that in normal JZSMCS CB1 and PCNA messenger RNA (mRNA) and protein expression was significantly higher in the proliferative phase of the menstrual cycle than in the secretory phase. CB1 and PCNA expression in JZSMCs from women with ADS was significantly higher than that in control women and did not significantly differ across the menstrual cycle. CB1 receptor antagonist AM251 inhibited the proliferation of adenomyotic JZSMCs in a dose-dependent manner. The CB1 receptor agonist ACEA significantly promoted the proliferation of adenomyotic JZSMCs. The apoptosis rate of adenomyotic JZSMCs treated with AM251 was significantly higher than that of JZSMCs from the untreated control group. The apoptosis rate was significantly decreased in the ACEA group compared with that in the untreated control group. Furthermore, AM251 suppressed the phosphorylation of AKT and Erk1/2 in adenomyotic JZSMCs. The CB1 agonist ACEA significantly promoted the phosphorylation of AKT and Erk1/2.

Conclusions: Our results indicated that the levels of CB1 and PCNA were increased in patients with adenomyosis and that cyclic changes were lost. CB1 may affect uterine JZ proliferation and apoptosis in adenomyosis by enhancing AKT and MAPK/Erk signalling.

Keywords: Adenomyosis, Junctional zone, Cannabinoid receptor, CB1, Proliferation, Apoptosis

\footnotetext{
* Correspondence: duanhuascience@163.com

Department of Minimally Invasive Gynecologic Center, Beijing Obstetrics and

Gynecology Hospital, Capital Medical University, 100006 Beijing, China
}

(C) The Author(s). 2021 Open Access This article is licensed under a Creative Commons Attribution 4.0 International License, which permits use, sharing, adaptation, distribution and reproduction in any medium or format, as long as you give appropriate credit to the original author(s) and the source, provide a link to the Creative Commons licence, and indicate if changes were made. The images or other third party material in this article are included in the article's Creative Commons licence, unless indicated otherwise in a credit line to the material. If material is not included in the article's Creative Commons licence and your intended use is not permitted by statutory regulation or exceeds the permitted use, you will need to obtain permission directly from the copyright holder. To view a copy of this licence, visit http://creativecommons.org/licenses/by/4.0/ The Creative Commons Public Domain Dedication waiver (http://creativecommons.org/publicdomain/zero/1.0/) applies to the data made available in this article, unless otherwise stated in a credit line to the data. 


\section{Introduction}

Adenomyosis (ADS) is a common oestrogen-dependent uterine disorder distinguished by non-malignant invasion of the bioactive endometrium into the myometrial wall, and it can result in abnormal uterine bleeding, dysmenorrhoea, and subfertility [1, 2]. Junctional zone (JZ) dysfunction has been suggested as the causative factor in the development of ADS [3]. On T2-weighted magnetic resonance imaging (MRI) of the uterus, the JZ myometrium can be clearly distinguished from the endometrium and outer myometrium, and diffuse or focal thickening of this zone to a thickness greater than $12 \mathrm{~mm}$ has become the most widely accepted criterion for establishing the presence of ADS [4]. Our previous studies also found that in ADS the JZ showed cellular and nuclear hypertrophy, abnormal nuclear and mitochondrial morphology, abundant myelin bodies and intermediate filament aggregates, extensive endoplasmic reticulum, and lengthening of sarcolemma plaques [5]. However, the specific mechanism of the abnormal proliferation of JZ smooth muscle cells (JZSMCs) remains unknown.

Endocannabinoids were first discovered in the early 1990s [6]. The endocannabinoid system (ECS) comprises endogenously produced bioactive lipids, their molecular targets (two well-characterized G-protein-coupled cannabinoid receptors, CB1 and CB2), synthesis and degradation enzymes, and protein transporters $[7,8]$. In recent years, the ECS has been reported to be involved in processes relevant to endometriosis, including cell migration, cell proliferation, apoptosis, and inflammation, and to interact with sex steroid hormones $[9,10]$. However, such data on ADS are sparse. A large number of studies have confirmed that $\mathrm{CB} 1$ can affect the proliferation, migration and apoptosis of many types of cells by regulating the AKT and MAPK/Erk pathways (via phosphorylation activation) $[11,12]$. In our previous study, we confirmed that expression of the cannabinoid receptors CB1 and CB2 was significantly higher in the ADS myometrium than in the normal myometrium, and CB1 expression in the JZ was positively correlated with the severity of dysmenorrhoea in patients with ADS [13]. However, whether the abnormally increased expression of CB1 is related to thickening of the JZ has not been reported. In addition, whether the increased activation of CB1 causes abnormalities in proliferation and apoptosis signalling is not entirely understood.

Based on these findings, it is reasonable to hypothesize that $\mathrm{CB1}$ regulates proliferation in the JZ and participates in the pathogenesis of ADS. This study investigated the expression of $\mathrm{CB} 1$ and proliferating cell nuclear antigen (PCNA) in cultured JZSMCs from women with and without ADS. Furthermore, we observed the effect of $\mathrm{CB} 1$ on the proliferation and apoptosis of JZSMCs and related signalling molecules through pharmacological intervention of CB1 receptors.

\section{Methods \\ Patients and specimens}

The study was approved by the local research and ethics committee of the Beijing Obstetrics and Gynecology Hospital (No. 2016-KY-012). All patients included in this study signed an informed consent form. From March 2018 to July 2019, uterine samples were acquired at Beijing Obstetrics and Gynecology Hospital from two groups or patients. The control group consisted of 17 premenopausal women who underwent hysterectomy due to cervical intraepithelial neoplasm III (CIN III) at Beijing Obstetrics and Gynecology Hospital. Clinical examination of the control subjects confirmed regular menstruation with no evidence of ADS. The study group consisted of 21 premenopausal women who reported dysmenorrhoea or other symptoms of adenomyosis, and their histopathologic examination had been confirmed as ADS. Samples of the JZ were obtained as soon as the uterus was removed during surgery. Based on the reported thickness of the JZ in normal reproductive women [4], we took samples from $2 \mathrm{~mm}$ beneath the endometrium of the JZ.

All patients had normal menstrual cycles; no evidence of endometriosis, endometrial pathology or malignancy; and no history of intrauterine device placement or hormone therapy within three months before surgery. The ADS group consisted of 11 women in the proliferative phase and 10 women in the secretory phase, and the control group consisted of 10 women in the proliferative phase and 7 women in the secretory phase. The mean (standard deviation [SD]) ages of women in the case and control groups were $44.5(\mathrm{SD}=4.3$, range $=38-49)$ and $43.2(\mathrm{SD}=$ 3.6 , range $=39-47)$ years, respectively. No significant difference in either age $(P=0.25)$ or menstrual cycle $(P>0.99)$ existed between the two groups. Cycle phase assignment was confirmed by endometrial histological assessment. The details are shown in Table 1.

\section{Cell culture and drug treatment}

Fresh junctional myometrium was obtained from the uterine samples within $10 \mathrm{~min}$ of surgical removal and opened in the sagittal plane. The tissue was collected in ice-cold saline, washed, and processed for cell dispersion. The endometrium was removed by gentle scratching at the endometrial-myometrial interface (EMI) with a surgical blade. Human uterine JZSMCs were cultured and identified as previously described, and cells at passages 2 to 6 were used [14-16]. To prevent contact inhibition or promotion, cells at no more than $75 \%$ 
Table 1 Overview of the demographics and other characteristics of the recruited patients

\begin{tabular}{llll}
\hline Parameter & Adenomyosis & Control & $\mathbf{N = 1 7}$ \\
\hline Ag= mean (SD) & $44.5(4.3)$ & $43.2(3.6)$ & $P$ \\
Menstrual cycle (Proliferative phase) $\mathrm{n}(\%)$ & $11(52.4 \%)$ & $10(58.8 \%)$ & $P=0.25$ \\
Uterine fibroids n (\%) & $8(38.1 \%)$ & $4(23.5 \%)$ & $P>0.99$ \\
Gravidity, median (range) & $2(0,5)$ & $2(1,5)$ & $P>0.53$ \\
Parity, median (range) & $1(0,2)$ & $1(0,2)$ & $P=0.75$ \\
PBAS, mean (SD) & $158.7(67.6)$ & $112.9(25.9)$ & $P=0.59$ \\
Chronic medication n & 0 & 0 & $P=0.12$ \\
Previous uterine surgery $n$ & 0 & 1 & $P>0.99$ \\
\hline
\end{tabular}

confluence were starved of endogenous steroids for 24 hours in phenol red-free Dulbecco's modified Eagle medium (Gibco, Grand Island, New York) supplemented with $2 \%$ charcoal-stripped foetal bovine serum (Biosera, Kansas City, Missouri). Then, cells in "starvation medium" were exposed to the following compounds: the CB1 receptor antagonist AM251 (Sigma-Aldrich, St. Louis, Missouri), and the CB1 receptor agonist ACEA (Sigma-Aldrich).

\section{Immunohistochemistry}

Immunohistochemistry was performed as described previously [17]. Sections $(4 \mu \mathrm{m})$ were prepared from each sample, dewaxed in xylene, rehydrated with a graded ethanol series and rinsed in distilled water. For antigen retrieval, the sections were boiled in citric saline (10 mmol/L, pH 6.0) for half an hour. Then, the samples were treated with a $3 \%$ hydrogen peroxide solution for 25 min to block endogenous peroxidase activity. After the samples were blocked with $3 \%$ bovine serum albumin (BSA, Servicebio, Wuhan, China) for $30 \mathrm{~min}$ at room temperature, they were incubated with either rabbit anti-CB1 antibody (Cell Signaling Technology, diluted 1:300) or mouse anti-PCNA antibody (Cell Signaling Technology, diluted $1: 100$ ) at $4{ }^{\circ} \mathrm{C}$ overnight. For the negative controls, phosphate-buffered saline (PBS) was used instead. Next, the sections were rinsed in PBS 3 times and incubated with horseradish peroxidaselabelled goat anti-rabbit antibody (Servicebio; diluted 1: 200) for $50 \mathrm{~min}$ at room temperature. After being washed with PBS and incubated with 3,3'-diaminobenzidine tetrahydrochloride dihydrate (Servicebio), the sections were counterstained with haematoxylin for $3 \mathrm{~min}$. Finally, all slides were mounted on glass slides with Permount (Servicebio), examined with a Leica DM4000B microscope (Leica, Wetzlar, Germany) and imaged with Leica Application Suite (LAS, version 4.9.0, Leica). The immunohistochemical staining results were assessed with Image-Pro Plus 6.0 software (Media Cybernetics, Rockville, Maryland) as reported previously [18]; the evaluators were blinded to the patients' information. A series of 10 images of each section for each targeted protein were randomly extracted to obtain an average value for statistical comparison. Colour intensity was used to define the staining intensity, and a colour mask was generated. Then, the mask was equally applied to all images, and measurements were acquired. The mean optical density (MOD), defined as the ratio of the integrated optical density (IOD) to the total stained area, was recorded and considered to be equivalent to the immunoreactivity level of the target substance in the endometrium.

\section{Immunoblotting}

JZSMCs were harvested, counted, and lysed in ice-cold Frackelton buffer [10 mM Tris- $\mathrm{HCl}$ (pH 7.05), $50 \mathrm{mM}$ $\mathrm{NaCl}, 30 \mathrm{mM}$ sodium pyrophosphate, $50 \mathrm{mM} \mathrm{NaF}, 1 \%$ Triton X-100, $1 \mathrm{Na}_{3} \mathrm{VO}_{4}$, one protease inhibitor cocktail tablet (Roche), and $1 \mathrm{mM}$ phenylmethylsulfonyl fluoride]. Insoluble material was removed by centrifugation of the samples at $20,000 \times g$ for $20 \mathrm{~min}$ at $4{ }^{\circ} \mathrm{C}$. Cytosolic and nuclear cellular lysates were prepared using NE-PER Nuclear and Cytoplasmic Extraction Reagents from a kit (Pierce Chemical Co., Colfax, CA, USA) according to the manufacturer's protocol. The protein concentrations in the total-cell lysates and cytosolic or nuclear extracts were determined using the bicinchoninic acid protein assay (Thermo Scientific, IL, USA). Twenty micrograms of each normalized sample was immunoblotted as previously described ${ }^{9}$ and probed with the following primary antibodies: anti-CB1, anti-PCNA (BD Biosciences, CA, USA), anti-AKT, anti-p-AKT (Santa Cruz Biotechnology, Santa Cruz, CA, USA), anti-Erk1/2, anti-p-Erk1/2 (Cell Signalling Technology, Danvers, MA, USA), and anti- $\beta$ actin (Sigma-Aldrich). The blots were then incubated with HRP-conjugated secondary antibody (Pierce Chemical Company, Rockford, IL, USA) for 1 hour at room temperature. Detection of the reactive antigens was performed with an ECL kit (Amersham Life Sciences, Inc., Arlington Heights, IL, USA), followed by exposure of 
the membrane to X-ray film. The resulting images were analysed with a ChemiImager 4000 system (Alpha Innotech Corporation, San Leandro, CA, USA) to determine the integrated density value (IDV) for each protein band normalized to the IDV for $\beta$-actin. All data are presented as the mean of three independent experiments.

\section{Quantitative real-time polymerase chain reaction ( $q R T-P C R$ )}

Total RNA was extracted from the JZSMCs with RNAiso Plus (Takara Bio, Inc., Shiga, Japan) and quantified with a NanoDrop 2000/2000c spectrophotometer (Thermo Fisher Scientific Inc., Massachusetts, USA). A PrimeScriptTM RT Reagent Kit (RR047A, Takara) was used to synthesize cDNA from $1 \mu \mathrm{g}$ of total RNA per sample. The primers used in this study were designed by Sangon Biotech Co., Ltd. (Shanghai, China), and their sequences are presented in Table 2. PCR was performed on an ABI 7500 real-time polymerase chain reaction system (Applied Biosystems, Grand Island, New York) with the protocol for SYBR Premix Ex TaqTM II (RR820A, Takara). The reaction protocol consisted of $95{ }^{\circ} \mathrm{C}$ for 30 seconds for initial denaturation followed by 40 cycles of 5 seconds at $95{ }^{\circ} \mathrm{C}$ and 34 seconds at $60{ }^{\circ} \mathrm{C}$. The $2^{-\triangle \mathrm{CT}}$ method was used to analyse relative gene expression as reported ${ }^{19}$ in which $\Delta \mathrm{CT}=\mathrm{CT}$ (target gene) $-\mathrm{CT}$ (internal control).

\section{CCK-8 assays}

CCK-8 (Dojindo Technology Co., Ltd.) assays were used to assess cell proliferation and cell viability. JZSMCs were cultured in 96-well plates at a density of 4,000 cells/well and incubated at $37{ }^{\circ} \mathrm{C}$ in $5 \% \mathrm{CO}_{2}$. CCK- 8 solution $(10 \mu \mathrm{l})$ was added to each well at different times and incubated for 4 hours, after which the plate was shaken for $1 \mathrm{~min}$. Finally, the absorbance at $450 \mathrm{~nm}$ was measured with a microplate reader.

\section{Flow cytometry assay}

After serum starvation followed by inhibitor or agonist treatment, cells seeded in 6-cm dishes were harvested and fixed in ice-cold $70 \%$ ethanol at $4{ }^{\circ} \mathrm{C}$ overnight. On the following day, fixed cells were incubated in $500 \mathrm{~mL}$ of propidium iodide/RNase staining buffer (BD Biosciences, San Jose, California) for $15 \mathrm{~min}$ in the dark at room temperature. The stained cells were then analysed using a

Table 2 Specific primers used for qRT-PCR analysis

\begin{tabular}{lll}
\hline Gene & & Sequence \\
\hline CB1 & Forward & 5'-CCTAGATGGCCTTGCAGATACC-3' \\
& Reverse & 5'-GAATGTCATTTGAGCCCACGTA-3' \\
PCNA & Forward & 5'-TTCGA ACCTACCGCTGC-3' \\
& Reverse & 5'-TCTCCTGGTTTGGTGCTC-3' \\
\multirow{3}{*}{-actin } & Forward & 5'-TGCCGACAGGATGCAGAAG-3' \\
& Reverse & 5'-CTCAGGAGGAGCAATGATCTTGA-3' \\
\end{tabular}

Gallios flow cytometer (Beckman Coulter, Brea, California). Cell debris and fixation artefacts were gated out, and the percentage of cells in each phase of the cell cycle was quantified using ModFit 4.0 software. At least 10,000 cells in each sample were analysed to obtain a measurable signal.

\section{Statistical analysis}

The statistical analysis was performed using Statistical Product and Service Solutions (SPSS) 22.0. After the normality of the data distribution was verified by the Kolmogorov-Smirnov test, the mean \pm SD from three independent replicates of each experiment was calculated. The independent $\mathrm{t}$-test was used to compare two groups. One-way analysis of variance (ANOVA) was applied to assess the statistical significance of differences between more than two groups. Before ANOVA or the independent $\mathrm{t}$-test, homogeneity of variance between groups was analysed using the $\mathrm{F}$ test. Differences for which $\mathrm{P}<0.05$ were considered statistically significant.

\section{Results \\ CB1 and PCNA protein expression levels in the adenomyotic JZ}

PCNA is an important nuclear marker of cell proliferation. In the ADS and control groups, PCNA and CB1 expression was observed in endometrial glandular epithelial cells, stromal cells, vascular endothelial cells, JZSMCs and ectopic endometrial cells (Fig. 1a). Brown PCNA- and CB1positive granules were observed in the cytoplasm and cell nuclei. Immunohistochemical staining was preliminarily performed to investigate the expression of $\mathrm{CB} 1$ and PCNA in the JZ myometrium in the two groups. The MOD of CB1 and PCNA was significantly increased in the adenomyotic JZ compared with that in the normal JZ (for the proliferative phase, both $P<0.001$; for the secretory phase, both $P<0.001)$. CB1 and PCNA immunoreactivity in the control JZ was higher in the proliferative phase of the menstrual cycle than in the secretory phase $(P<0.05)$. However, cyclic variation in CB1 and PCNA immunoreactivity was not observed in the adenomyotic JZ $(P=0.087$ and $P=0.916$, respectively) (Fig. $1 \mathrm{~b}$ and c).

\section{CB1 and PCNA protein expression levels in adenomyotic JZSMCs}

The Western blotting results showed no significant differences in CB1 or PCNA protein expression in JZSMCs in the ADS group between the proliferative and secretory phases $(P>0.05)$. In the control group, the expression levels of $\mathrm{CB} 1$ and PCNA in the proliferative phase were significantly greater than those in the secretory phase $(P<0.05)$. The CB1 and PCNA levels in both the proliferative and secretory phases were higher in the JZSMCs of the ADS group than in those of the control group (for the proliferative phase, $P<0.05, P<$ 

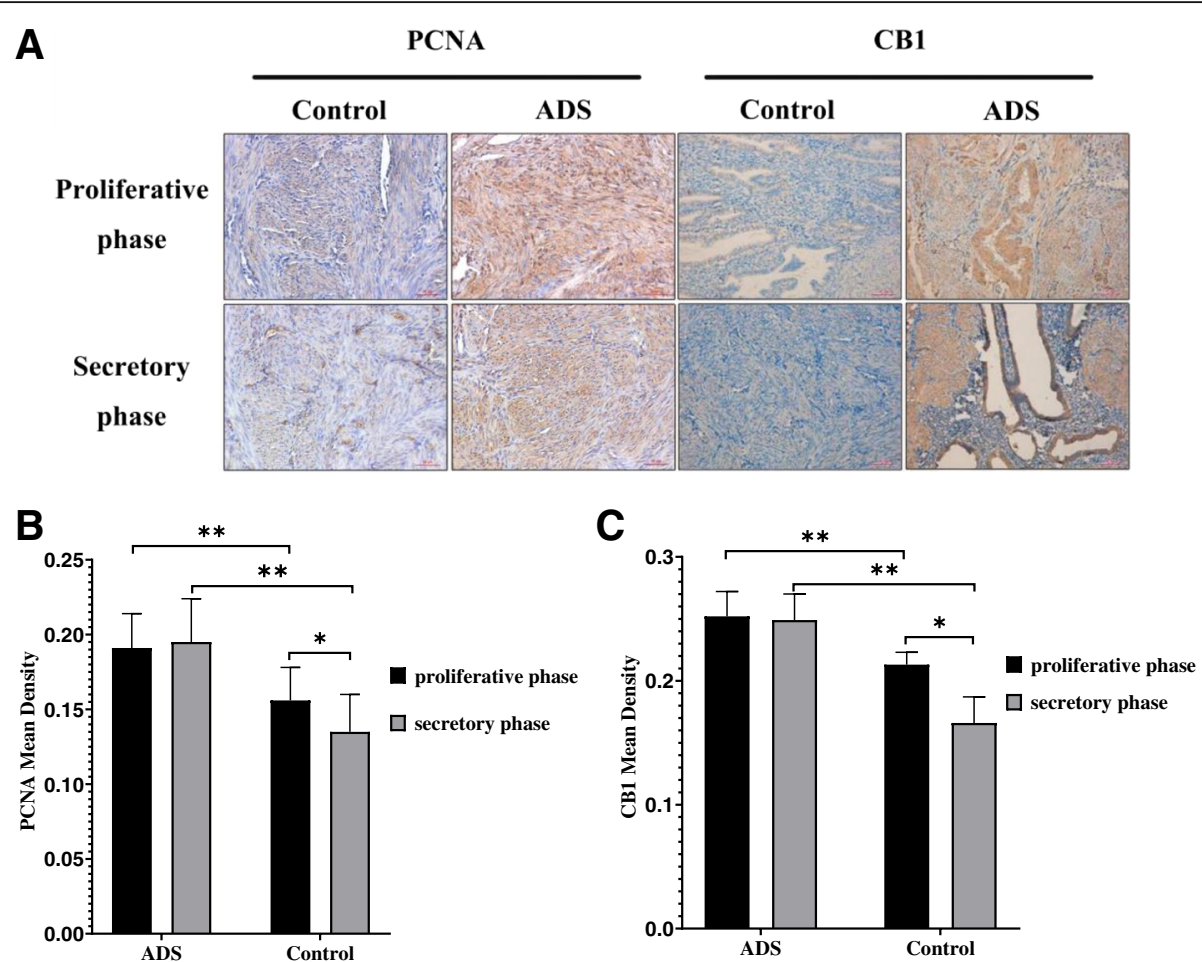

Fig. 1 Immunohistochemical staining for CB1 and PCNA. a CB1 and PCNA expression in women in the adenomyosis group and control group in the proliferative and secretory phases. $\mathbf{b}$ Quantitative analysis of the mean optical density (MOD) of CB1 between the proliferative phase and secretory phase in the adenomyotic JZ and control JZ. c Quantitative analysis of the mean optical density (MOD) of CB1 between the proliferative phase and secretory phase in the adenomyotic JZ and control JZ. All micrographs are magnified 200x. Scale bars represent $5 \mu \mathrm{m}$. The error bars on all histograms indicate the standard deviation. ${ }^{*}, P<0.05 ;{ }^{*}, P<0.01$

0.01 , respectively; for the secretory phase, $P<0.05, P<$ 0.01 , respectively) (Fig. $2 \mathrm{a}$ and $\mathrm{b}$ ).

\section{CB1 and PCNA mRNA expression levels in adenomyotic JZSMCs}

To further determine the CB1 and PCNA expression levels in the JZSMCs of women with ADS and control women, we observed the CB1 and PCNA messenger RNA (mRNA) expression levels using qRT-PCR and found them to be similar. In the control group, CB1 and PCNA mRNA expression was significantly higher in the proliferative phase than in the secretory phase $(P<0.05)$. In the ADS group, no significant difference in $\mathrm{CB} 1$ or PCNA mRNA expression between the secretory and proliferative phases was observed $(P>0.05)$. The comparison of CB1 and PCNA mRNA expression in JZSMCs showed significantly higher transcript levels in the ADS group than in the control group $(P<0.05)$ (Fig. 3a and b).

\section{Effect of pharmacological intervention of the CB1 receptor on the proliferation of JZSMCs in the adenomyotic uterus}

To elucidate the role of CB1 in the proliferation of human uterine JZSMCs, we stimulated and inhibited the activity of CB1 in adenomyotic JZSMCs and then detected their capacity for cell proliferation. The CCK-8 assay showed that the CB1 receptor antagonist AM251 inhibited the proliferation of adenomyotic JZSMCs in a dose-dependent manner. The cell survival rate was significantly decreased by AM251 at all concentrations in the physiological range that were tested $\left(10^{-8} \mathrm{~mol} / \mathrm{L}\right.$, $10^{-7} \mathrm{~mol} / \mathrm{L}, 10^{-6} \mathrm{~mol} / \mathrm{L}$, and $\left.5 \times 10^{-6} \mathrm{~mol} / \mathrm{L}\right)(P<0.05$, Fig. 4a). The $\mathrm{IC}_{50}$ of AM251 in the JZSMCs was $6.931 \times$ $10^{-7} \mathrm{~mol} / \mathrm{L}$, as shown by regression analysis with GraphPad Prism 6.0 software; thus, the optimal concentration of AM251 used in the following experiment was $10^{-6}$ mol/L (Fig. 5b).

The CB1 receptor agonist ACEA promoted the proliferation of JZSMCs in the ADS group. After treatment with ACEA at different concentrations $\left(10^{-7} \mathrm{~mol} / \mathrm{L}\right.$, $10^{-6} \mathrm{~mol} / \mathrm{L}$ and $\left.5 \times 10^{-6} \mathrm{~mol} / \mathrm{L}\right)$, the cell survival rate increased with increasing ACEA concentration; meanwhile, the cell survival rate at $10^{-5} \mathrm{~mol} / \mathrm{L}$ was significantly lower than that of the group treated with $5 \times 10^{-6}$ mol/L ACEA $(P<0.01$, Figs. 3, 4, 5 and $6 \mathrm{c})$. Therefore, the optimal concentration of ACEA used in the following experiment was $5 \times 10^{-6} \mathrm{~mol} / \mathrm{L}$ (Fig. 4c).

To further clarify the role of the CB1 receptor, adenomyotic JZSMCs were divided into four groups for 

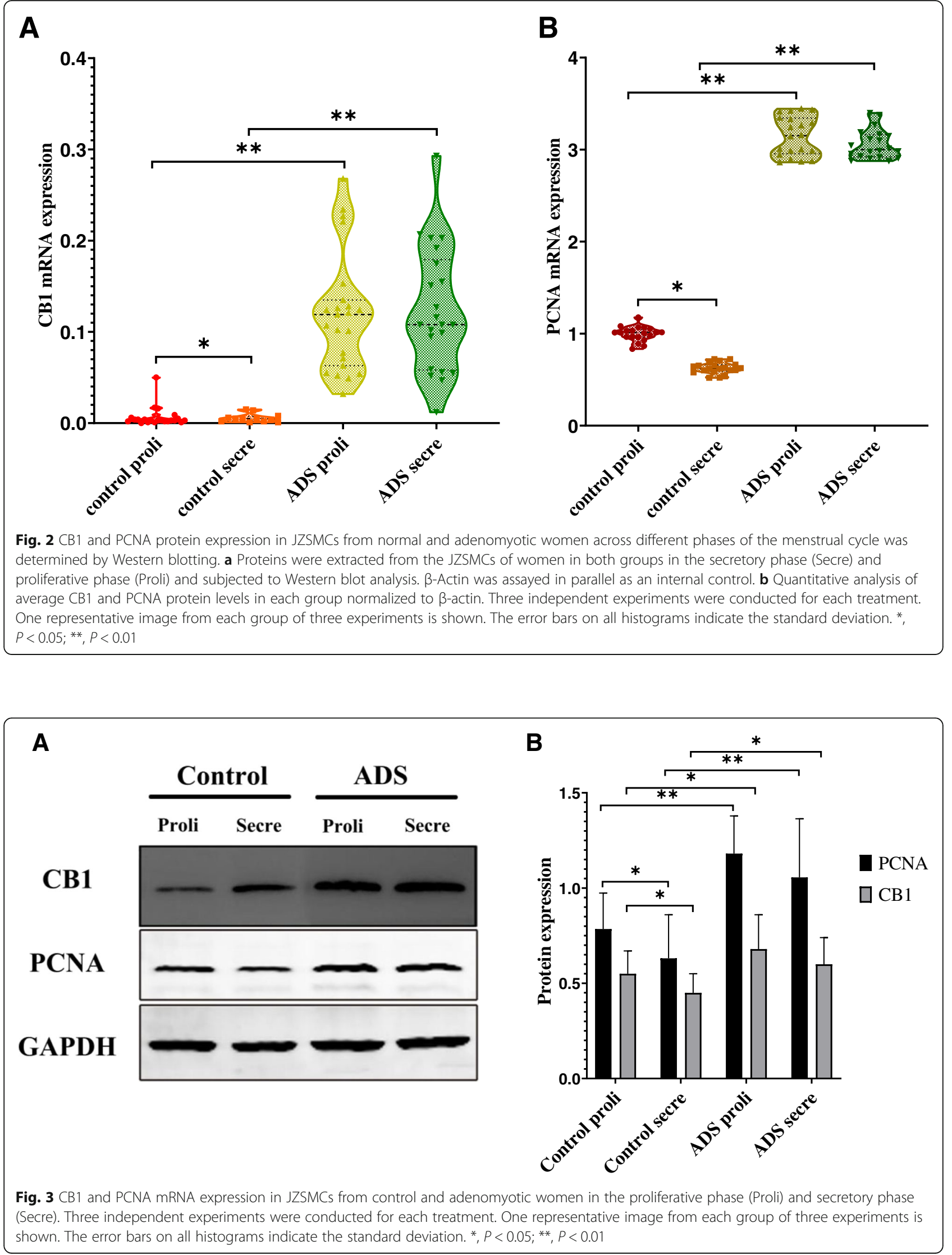

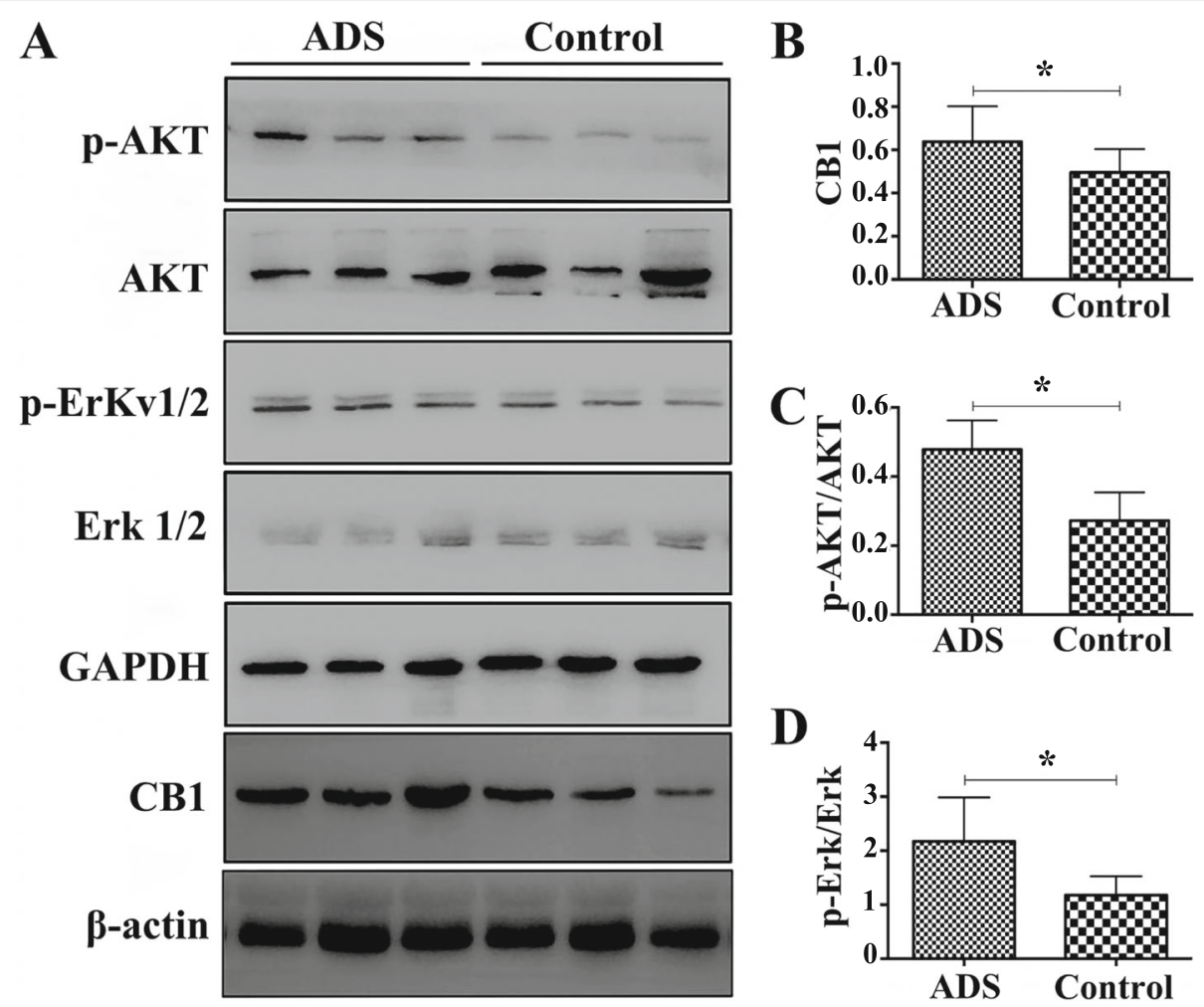

Fig. 4 Expression of the CB1, AKT, p-AKT, Erk1/2 and p-Erk1/2 proteins in JZSMCs. a Characteristic blots for each protein. b Comparison of intercellular CB1 protein expression in each group. c Comparison of the intercellular p-AKT/AKT ratios. $\mathbf{d}$ Comparison of the intercellular p-Erk/Erk ratios. Three independent experiments were conducted for each treatment, and one representative image from each group of three experiments is shown. The error bars on all histograms indicate the standard deviation. ${ }^{*}, P<0.05$

pharmacological interference with the CB1 receptor. The four groups were pretreated with serum-free medium containing phenol red (control group), $10^{-6} \mathrm{~mol} / \mathrm{L}$ AM251 (AM251 group), $5 \times 10^{-6} \mathrm{~mol} / \mathrm{L}$ ACEA (ACEA group) or $10^{-6} \mathrm{~mol} / \mathrm{L}$ AM251 for $30 \mathrm{~min}$ followed by $5 \times$ $10^{-6} \mathrm{~mol} / \mathrm{L}$ ACEA (combination group). The cell survival rate of the control group was set as $100 \%$. The cell survival rate in the ACEA group was $169.9 \pm 18.3 \%$, which was significantly higher than that in the control group $(P<$ $0.001)$. The cell survival rate in the AM251 group was $41.5 \pm 3.5 \%$, which was significantly lower than that in the control group $(P<0.001)$. No significant difference in cell survival rate was found between the combination group and the control group $(P>0.05)$ (Fig. 5d).

\section{Effect of pharmacological intervention of the CB1} receptor on JZSMC apoptosis in adenomyotic uteri

A flow cytometry assay showed that the apoptosis rate of JZSMCs after treatment with AM251 was significantly higher in the ADS group than in the control group $(P<0.001)$. The apoptosis rate in the ACEA group was significantly lower than that of the control group $(P<0.001)$. No significant difference in apoptosis rate was found between the combination group and the control group $(P>0.05)$ (Fig. 5a and b).

Evidence for the involvement of CB1 in activation of the AKT/Erk pathway in adenomyotic JZSMCs

The AKT signalling pathway plays a vital role in regulating cell progression and inhibiting proapoptotic protein expression. We aimed to investigate whether CB1 is involved in the activation of AKT in adenomyotic JZSMCs. Immunoblotting showed that the CB1 antagonist AM251 suppressed the phosphorylation of $\mathrm{AKT}$ and Erk1/2 in adenomyotic JZSMCs. The CB1 receptor agonist ACEA significantly promoted the phosphorylation of AKT and Erk1/2. The expression of CB1 and the phosphorylation of AKT and Erk $1 / 2$ did not significantly differ between the combination group and control group, which further suggested that $\mathrm{CB} 1$ is involved in activation of the AKT/Erk pathway (Fig. 7a-d).

\section{Discussion}

Abnormal thickening of the JZ can influence sperm transport and embryo implantation and increase intrauterine pressure, resulting in infertility, dysmenorrhoea 


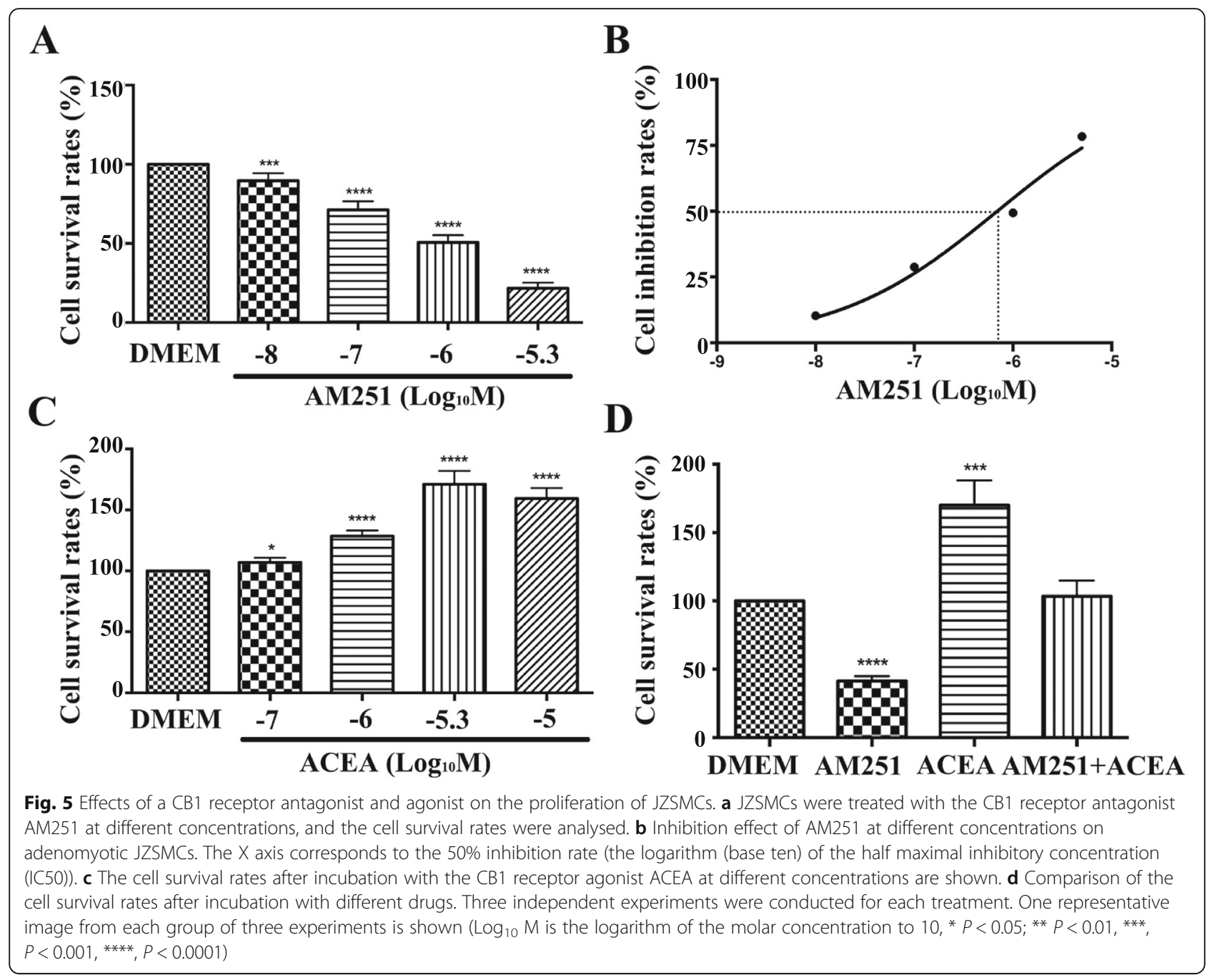

and menorrhagia. $[19,20]$. Our previous electron microscopic research showed that adenomyotic JZSMCs exhibit hyperplasia, and analysis of the ultrastructure indicated the loss of cyclical changes [5]. Exploration of the specific manifestations and mechanism of proliferation dysfunction in JZSMCs is of great importance in uncovering the pathogenesis of ADS. PCNA is an important nuclear marker of cell proliferation [21]. In this study, we first detected PCNA expression in the JZ from specimens obtained from both ADS and non-ADS patients to reveal the proliferative characteristics of JZ. PCNA showed persistently high expression in the JZ, and cyclical changes were lost, which may be related to irregular thickening in ADS.

In our study, the proliferative capacity of JZSMCs in the ADS group was higher than that in the control group, which also explained the abnormal thickening of the JZ observed in ADS. Through molecular biology technology, we further demonstrated that CB1 expression in JZSMCs differed between the ADS and control groups; the protein and gene expression of CB1 was significantly higher in the ADS group than in the control group. The adenomyotic uterus has been previously reported to exhibit obvious myocyte hypertrophy, in which cytoplasmic aggregates were formed by abundant intermediate filaments, the edge of the nucleus was clear, the nucleolus and surrounding nuclear chromatin were obvious, and the rough endoplasmic reticulum and Golgi complex were more prominent than those in the absence of ADS, indicating that protein synthesis was more active; all of these characteristics were more pronounced in the JZ [22]. This may be the material basis for CB1 upregulation as well as the theoretical basis for the experimentally observed JZSMC hypertrophy in the ADS group. In 2004, Dennedy et al. [23] demonstrated the presence of the cannabinoid receptors CB1 and CB2 in human uterine smooth muscle for the first time. They studied myometrial biopsy specimens from caesarean section at term and found that both endogenous and exogenous cannabinoids could mediate the relaxation of 

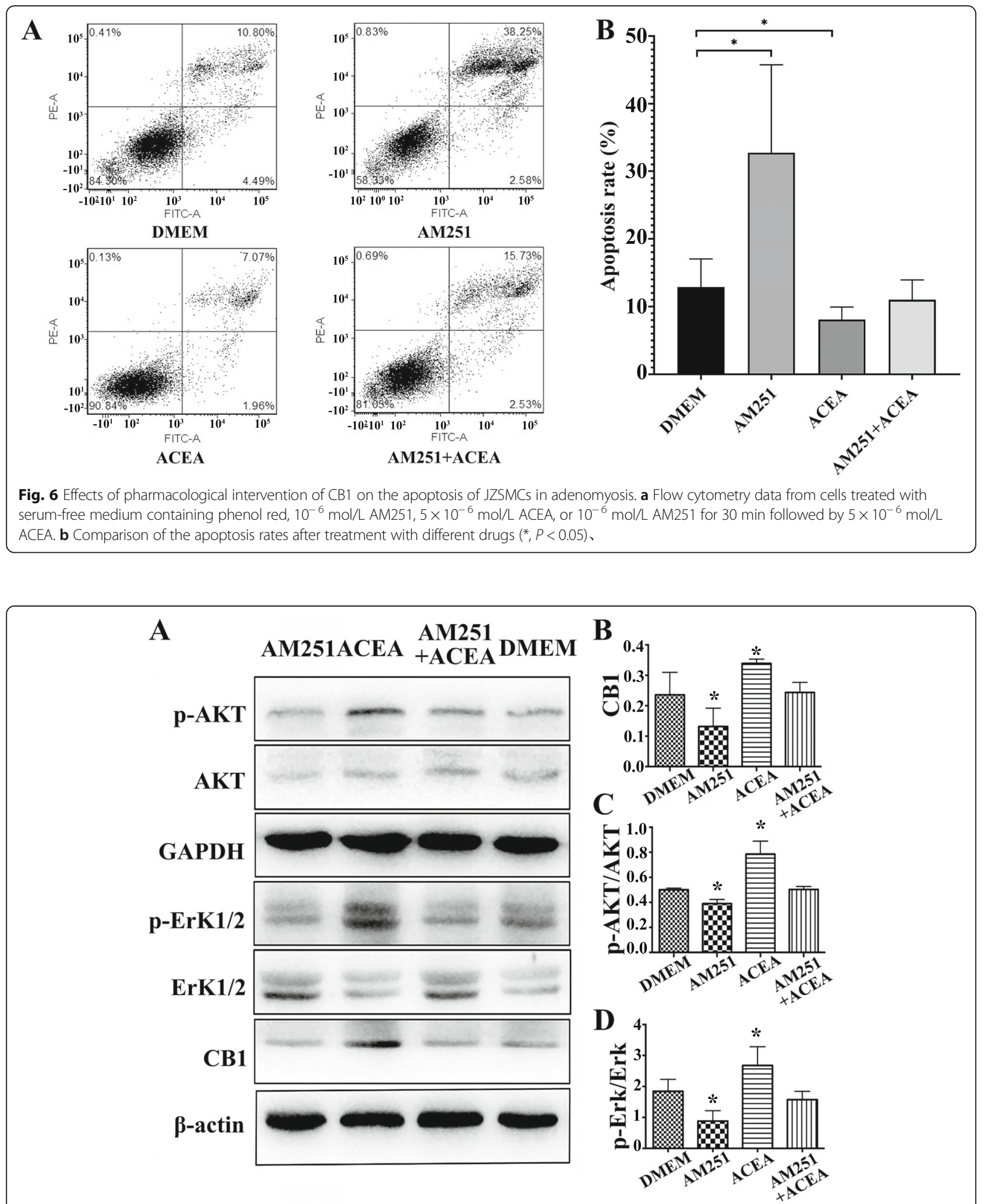

Fig. 7 Effect of pharmacological intervention of CB1 on expression of the CB1, AKT,p-AKT, Erk1/2 and p-Erk1/2 proteins in adenomyotic JZSMCs. a Characteristic blots foreach protein. $\mathbf{b}$ Comparison of intercellular CB1 protein expression in each group. c Comparison of the intercellular pAKT/AKT ratios. $\mathbf{d}$ Comparison of the intercellularp-Erk/Erk ratios. Three independent experiments were conducted for each treatment, and one representative image from each group of three experiments is shown. The error bars on all histograms indicate the standard deviation. ${ }^{*}, P<0.05$ 
human uterine smooth muscle in vitro during pregnancy by the $\mathrm{CB} 1$ receptor rather than the $\mathrm{CB} 2$ receptor. Uterine leiomyoma and ADS are oestrogen-dependent diseases, and a recent study showed no significant difference in CB1 expression between fibroid tissue and the normal myometrium, which seems inconsistent with our findings. However, the specimens used in that report were from patients aged 37 to 77 years, which was not appropriate for the study of uterine fibroids. For endometriosis, which exhibits pathological characteristics similar to those of ADS, studies have shown that activation of CB1 could promote the growth of lesions in endometriosis animal models and the migration of endometrial stromal cells [24-27]. A study also reported that the expression of endogenous cannabinoids and their related intermediates was significantly increased in ADS patients during the menstrual cycle [26], which is consistent with our finding that the selective CB1 receptor agonist ACEA promoted the proliferation of JZSMCs.

We observed the expression levels of AKT and MAPK/Erk pathway proteins in the ADS and control groups by Western blotting to further identify the cause of differences in the in vitro proliferative capacity of the two groups. The total AKT and total Erk1/2 expression in the ADS group was comparable to that in the control group, but the expression of p-AKT and p-Erk $1 / 2$ significantly differed. The analysis revealed that both the $\mathrm{p}$ AKT/AKT and p-Erk/Erk ratios in the ADS group were significantly higher than those in the control group, suggesting abnormal activation of the AKT and MAPK/Erk pathways in the abnormally proliferating cells of the ADS group.

Studies have shown that activation of the AKT and MAPK/Erk pathways not only promotes the proliferation and migration of endometrial stromal cells in endometriosis $[28,29]$ but also participates in the progesterone resistance of endometriosis [30], suggesting that the AKT and MAPK/Erk pathways are potential targets for endometriosis treatment. The current study demonstrates that the AKT and MAPK/Erk pathways are also abnormally activated in aberrantly proliferating adenomyotic JZSMCs, suggesting that the AKT and MAPK/ Erk pathways are therapeutic targets for ADS.

The CCK- 8 assay results showed that the CB1 receptor antagonist AM251 dose-dependently inhibited the proliferation of JZSMCs in uterine ADS in vitro. Decreased cell survival was observed with increasing antagonist concentration. When JZSMCs were pretreated with AM251 before treatment with ACEA, the cell survival rate was comparable to that of the untreated group, suggesting that AM251 can completely antagonize the pharmacological activation of CB1 by ACEA. This finding is essentially consistent with the result reported by Eckardt et al., who showed that AM251 could also completely block the effect of arachidonoyl ethanolamine or anandamide (AEA, an endocannabinoid) in human skeletal muscle cells [31].

In the present study, the rate of apoptosis was analysed using flow cytometry. The results demonstrated that the selective CB1 receptor antagonist AM251 could promote the apoptosis of JZ smooth muscle cells in uterine ADS and completely block the inhibitory effect of its agonist, ACEA. Similar to the role of the CB1 receptor in cell proliferation, previous studies on the role of the CB1 receptor in apoptosis have suggested that it plays a dual role in apoptosis. Caunt et al. [32] found that ACEA promoted the survival of nerve cells through upregulating expression of the apoptosis suppressor protein Bcl-2. However, a contradictory result was reported by Gurbuz et al. [33], who showed that AM251 induced cell apoptosis in A375 human melanoma cells through downregulating expression of the apoptosis suppressor proteins $\mathrm{Bcl}-2$ and survivin and upregulating the expression of Bax.

To further elucidate the specific mechanism of the $\mathrm{CB} 1$ receptor in the proliferation and apoptosis of JZSMCs, the selective CB1 receptor agonist ACEA and antagonist AM251 were used for pharmacological intervention of the CB1 receptor. ACEA activated the intracellular CB1 receptor and upregulated the expression of p-AKT and p-Erk1/2 while AM251 could completely antagonize the effect of ACEA, which suggested that the expression of CB1 and the p-AKT/AKT and p-Erk/Erk ratios were not significantly different from those of the untreated group after pretreatment with AM251 and the subsequent ACEA treatment. These results suggested that the CB1 receptor can act on JZSMCs by activating $\mathrm{AKT}$ and the MAPK/Erk signalling pathway and that the selective CB1 receptor antagonist AM251 has potential therapeutic applications for ADS.

As a preliminary study, this report has several possible limitations. First, this study is not based on a fairly large sample size of patients, especially when detecting the impact of $\mathrm{CB} 1$ on the function of JZ in different phases of the menstrual cycle in ADS. Second, we did not discover the relationship of CB1 with the clinical characteristics of adenomyosis (focal and diffused) and potential cytokines, immune cells, and other potential molecular pathways.

In conclusion, we confirmed that uterine JZSMCs in the ADS displayed significantly greater proliferative ability than those in the control group. The cannabinoid receptor $\mathrm{CB} 1$, the major receptor involved in endocannabinoid function, promoted the proliferation of JZSMCs in uterine ADS and inhibited apoptosis by activating the AKT and MAPK/Erk signalling pathways. Our study on the role of the CB1 in adenomyosis will be helpful for determining whether the endocannabinoid 
system could be a novel target for treating adenomyosis. However, further research is still needed to better clarify this system in ADS.

\section{Abbreviations}

ADS: Adenomyosis; JZ: Junctional zone; PCNA: Proliferating cell nuclear antigen; JZSMCs: JZ smooth muscle cells; mRNA: Messenger RNA; MRI: Magnetic resonance imaging; ECS: Endocannabinoid system; CIN III: Cervical intraepithelial neoplasm III; EMI: Endometrial-myometrial interface; qRT-PCR: Quantitative real-time polymerase chain reaction

\section{Acknowledgements}

This study was funded by a grant from the Natural Science Foundation of China (grant number 81571412) and the Beijing Municipal Administration of Hospital Clinical Medicine Development of Special Funding Support (grant number ZYLX201406) and supported by the Beijing Obstetrics and

Gynecology Hospital, Capital Medical University (grant number FCYY201920).

\section{Authors' contributions}

SW performed the laboratory experiments, analysed the data and wrote the manuscript; BHL edited the manuscript and performed the data analysis. HD developed the project and edited the manuscript. All authors read and approved the final manuscript. ZC G, XL and FQS collected the tissues and edited the manuscript.

\section{Funding}

This study was funded by a grant from the Natural Science Foundation of China (grant number 81571412) and the Beijing Municipal Administration of Hospital Clinical Medicine Development of Special Funding Support (grant number ZYLX201406) and supported by the Beijing Obstetrics and

Gynecology Hospital, Capital Medical University (grant number FCYY201920).

\section{Availability of data and materials}

All analysis results are displayed in the results. For specific experimental data, please contact the corresponding author.

\section{Ethics approval and consent to participate}

The study was approved by the local research and ethics committee of the Beijing Obstetrics and Gynecology Hospital (No. 2016-KY-012). All patients included in this study signed an informed consent form.

\section{Consent for publication}

All authors consented to publication.

\section{Competing interests}

The authors report no conflicts of interest.

Received: 11 May 2020 Accepted: 26 December 2020 Published online: 02 February 2021

\section{References}

1. Naftalin J, Hoo W, Pateman K, Mavrelos D, Holland T, Jurkovic D. How common is adenomyosis? A prospective study of prevalence using transvaginal ultrasound in a gynaecology clinic. Hum Reprod. 2012;27(12): 3432-9.

2. Leyendecker $\mathrm{G}$, Wildt $\mathrm{L}$. A new concept of endometriosis and adenomyosis: tissue injury and repair (TIAR). Horm Mol Biol Clin Investig. 2011;5(2):125-42.

3. Fusi L, Cloke B. Brosens.The uterine junctional zone. Best Pract Res Clin Obstet Gynaecol. 2006;20(4):479-91.

4. Tamai K, Koyama T, Umeoka S, et al. Spectrum of MR features in adenomyosis. Best Pract Res Clin Obstet Gynaecol. 2006;20(4):583-602

5. Zhang Y, Zhou L, Li TC, Duan Hua Yu, Pei, Wang HY. Ultrastructural features of endometrial-myometrial interface and its alteration in adenomyosis. Int J Clin Exp Pathol. 2014;7:1469-77.

6. Devane WA, Hanus L, Breuer A, et al. Isolation and structure of a brain constituent that binds to the cannabinoid receptor. Science. 1992;258:1946-9.

7. Balsevich G, Petrie GN, Hill MN. Endocannabinoids: Effectors of glucocorticoid signaling. FRONT NEUROENDOCRIN. 2017;47:86-108.
8. Lu H, Mackie K. An Introduction to the Endogenous Cannabinoid System. BIOL PSYCHIAT 2016, 79(7)

9. Fraguas-Sánchez Al, Torres-Suárez Al. Medical Use of Cannabinoids Drugs. 2018:78(16):1665-703.

10. Rapino C, Battista N, Bari M, Maccarrone M. Endocannabinoids as biomarkers of human reproduction. Human Reproduction Update. 2014;20(4):501-16.

11. El-Talatini MR, Taylor AH, Konje JC. The relationship between plasma levels of the endocannabinoid, anandamide, sex steroids, and gonadotrophins during the menstrual cycle. Fertil Steril. 2010;93(6):1989-96.

12. Schander JA, Correa F, Bariani MV, Blanco J, Cymeryng C, Jensen F, Wolfson ML, Franchi AM. A role for the endocannabinoid system in premature luteal regression and progesterone withdrawal in lipopolysaccharide-induced early pregnancy loss model[J]. Mol Hum Reprod. 2016;22(11):800-8.

13. Shen $X$, Duan H, Wang S, Hong W, Wang $Y$, Lin S. Expression of Cannabinoid Receptors in Myometrium and its Correlation With Dysmenorrhea in Adenomyosis. REPROD SCI. 2019;26(12):1618-25.

14. Asada H, Yamagata $Y$, Taketani $T$, et al. Potential link between estrogen receptor-alpha gene hypomethylation and uterine fibroid formation. Mol Hum Reprod. 2008;14(9):539-45.

15. Cuppoletti J, Malinowska DH, Chakrabarti J, Ueno R. Effects of lubiprostone on human uterine smooth muscle cells. Prostaglandins Other Lipid Mediat. 2008;86(14):56-60.

16. Tahara A, Tsukada J, Tomura Y, et al. Pharmacologic characterization of the oxytocin receptor in human uterine smooth muscle cells. Br J Pharmacol. 2000;129(1):131-9.

17. Lin J, Huo R, Xiao L, Zhu X, Xie J, Sun S, He Y, Zhang J, Sun Y, Zhou Z, Wu P, Shen B, Li D, Li N. A novel p53/microRNA-22/Cyr61 axis in synovial cells regulates inflammation in rheumatoid arthritis. Arthritis \& Rheumatology (Hoboken, N.J.). 2014; 66(1): 49-59.

18. Liu X, Nie J, Guo SW. Elevated immunoreactivity to tissue factor and its association with dysmenorrhea severity and the amount of menses in adenomyosis. Human reproduction (Oxford England). 2011;26(2):337-45.

19. Li J, Chung JPW, Wang S, Li T, Duan H. The Investigation and Management of Adenomyosis in Women Who Wish to Improve or Preserve Fertility. BIOMED RES INT. 2018:2018:1-12.

20. Wang S, Duan H, Zhang Y. Abnormal activation of RhoA/ROCKI Signaling in junctional zone smooth muscle cells of patients. Reprod Sci. 2016;11(3): 1011-9.

21. Wang S, Duan H, Zhang Y, Sun FQ. Abnormal Activation of RhoA/ROCK-I Signaling in Junctional Zone Smooth Muscle Cells of Patients With Adenomyosis. REPROD SCI. 2015;23(3):333-41.

22. Iuvone T, De Filippis D, Di Spiezio Sardo A, D'Amico A, Simonetti S, Sparice S, Esposito G, Bifulco G, Insabato L, Nappi C, Guida M. Selective CB2 upregulation in women affected by endometrial inflammation. Journal Of Cellular Molecular Medicine. 2008;12(2):661-70.

23. Dennedy D, Friel MC, Houlihan AM, Broderick DD, Smith VM, Morrison T. JJ. Cannabinoids and the human uterus during pregnancy. Am J Obstet Gynecol. 2004;190(1):2-9. discussion 3A

24. Sanchez A-M, Quattrone F, Pannese M, Ulisse A, Candiani M, Diaz-Alonso J, Velasco G, Panina-Bordignon P. The cannabinoid receptor CB1 contributes to the development of ectopic lesions in a mouse model of endometriosis. Hum Reprod. 2017:32(1):175-84.

25. Han H, Liang X, Wang J, Zhao Q, Yang M, Rong W, Zhang G. Cannabinoid receptor 1 contributes to sprouted innervation in endometrial ectopic growth through mitogen-activated protein kinase activation. Brain Res. 2017;1663:132-40.

26. Gentilini D, Besana A, Vigano P, Dalino P, Vignali M, Melandri M, Busacca M, Di Blasio AM. Endocannabinoid system regulates migration of endometrial stromal cells via cannabinoid receptor 1 through the activation of PI3K and ERK1/2 pathways. Fertility Sterility. 2010;93(8):2588-93.

27. Cui N, Wang C, Zhao Z, Zhang J, Xu Y, Yang Y, Hao G. The Roles of Anandamide, Fatty Acid Amide Hydrolase, and Leukemia Inhibitory Factor on the Endometrium during the Implantation Window. Frontiers in Endocrinology. 2017:8:268

28. Makker A, Goel MM, Das V, Agarwal A. PI3K-Akt-mTOR and MAPK signaling pathways in polycystic ovarian syndrome, uterine leiomyomas and endometriosis: an update. Gynecol Endocrinol. 2012;28(3):175-81.

29. Ngô C, Nicco C, Leconte M, Chéreau C, Arkwright S, Vacher-Lavenu M-C, Weill B, Chapron C, Batteux F. Protein kinase inhibitors can control the progression of endometriosis in vitro and in vivo. J Pathol. 2010;222(2):148-57. 
30. Leconte M, Nicco C, Ngô C, Chéreau C, Chouzenoux S, Marut W, Guibourdenche J, Arkwright S, Weill B, Chapron C, Dousset B, Batteux F. The mTOR/AKT inhibitor temsirolimus prevents deep infiltrating endometriosis in mice. Am J Pathol. 2011;179(2):880-9.

31. McKinnon BD, Kocbek V, Nirgianakis K, Bersinger NA, Mueller MD. Kinase signalling pathways in endometriosis: potential targets for non-hormonal therapeutics. Human Reproduction Update. 2016;22(3):382-403.

32. Caunt CJ, Sale MJ, Smith PD, Cook SJ. MEK1 and MEK2 inhibitors and cancer therapy: the long and winding road[]]. Nature Reviews Cancer. 2015;15(10): 577-92.

33. Gurbuz N, Asoglu MR, Ashour AA, Salama S, Kilic GS, Ozpolat B. A selective serotonin 5 -HT1B receptor inhibition suppresses cells proliferation and induces apoptosis in human uterine leiomyoma cells. Eur J Obstet Gynecol Reprod Biol. 2016;206:114-9.

\section{Publisher's Note}

Springer Nature remains neutral with regard to jurisdictional claims in published maps and institutional affiliations.

Ready to submit your research? Choose BMC and benefit from:

- fast, convenient online submission

- thorough peer review by experienced researchers in your field

- rapid publication on acceptance

- support for research data, including large and complex data types

- gold Open Access which fosters wider collaboration and increased citations

- maximum visibility for your research: over $100 \mathrm{M}$ website views per year

At BMC, research is always in progress.

Learn more biomedcentral.com/submissions 